\title{
A Second-Order Uniformly Stable Explicit Asymmetric Discretization Method for One-Dimensional Fractional Diffusion Equations
}

\author{
Lin Zhu (D) \\ Ningxia University, School of Mathematics and Statistics, Yinchuan 750021, China \\ Correspondence should be addressed to Lin Zhu; xqdeng2002@163.com
}

Received 28 January 2019; Revised 6 May 2019; Accepted 14 May 2019; Published 28 May 2019

Academic Editor: Eulalia Martínez

Copyright (C) 2019 Lin Zhu. This is an open access article distributed under the Creative Commons Attribution License, which permits unrestricted use, distribution, and reproduction in any medium, provided the original work is properly cited.

Using the asymmetric discretization technique, an explicit finite difference scheme is constructed for one-dimensional spatial fractional diffusion equations (FDEs). The spatial fractional derivative is approximated by the weighted and shifted Grünwald difference operator. The scheme can be solved explicitly by calculating unknowns in the different nodal-point sequences at the odd time-step and the even time-step. The uniform stability is proven and the error between the discrete solution and analytical solution is theoretically estimated. Numerical examples are given to verify theoretical analysis.

\section{Introduction}

Fractional operator has been used to solve a certain class of problems arising from the real world [1-7]. Fractional spatial derivatives are suitable to describe anomalous dispersion and diffusion models. In particular, when the second-order derivative is replaced by the Riemann-Liouville fractional derivative of order $\alpha \in(1,2)$ in space, the fractional equation represents a superdiffusive flow model.

Up to now, several finite difference methods have been presented for the initial-boundary value problem of the onedimensional spatial fractional diffusion equations (FDEs) [815]. Because of the nonlocal property of fractional differential operators, many numerical methods for FDEs have full coefficient matrices which require storage of $O\left(K^{2}\right)$ and computational cost of $O\left(K^{3}\right)$ for a problem of size $K$, where $K$ is the number of discrete points in the spatial coordinate. How to save storage space and reduce the number of calculations is essential. It is well known that implicit scheme is more stable, but more complex in calculation than the explicit scheme.

The main purpose of this paper is to construct a semiimplicit finite difference scheme for solving one-dimensional spatial FDE by combining a second-order weighted and shifted Grünwald difference (WSGD) operator $[16,17]$ with the asymmetric discretization technique introduced by Saulyev [18]. Formally, the obtained finite difference scheme is implicit; however we can obtain the discrete solution of the scheme explicitly by using different nodal-point stencils at odd time-step and even time-step. Instead of solving the linear algebraic system, we compute the unknowns according to the sequences in left-to-right order at the odd time-step and then compute the unknowns according to the sequences in right-to-left order at the even time-step. We prove that the scheme is uniformly stable when $\alpha \in[(\sqrt{17}-1) / 2,2]$, and the error between the numerical and analytical solutions in discrete $l^{2}$ norm is of order $O\left(\Delta t^{2} h^{-2(\alpha-1)}+\Delta t+h^{2}\right)$, where $\alpha$ is the order of spatial fractional derivative, and $h$ and $\Delta t$ are the space and time mesh sizes. The error estimate here is better than that in [19], where the error in discrete $l^{2}$ norm is of order $O\left(\Delta t^{2} h^{-2(\alpha-1)}+\Delta t+h\right)$. Some examples are presented to show that the numerical results match our theoretical analysis.

This asymmetric technique has been used to construct parallel algorithms in several research results [20-23]. Within the scope of our knowledge, there are only two papers including this paper and the one Rui and Huang presented [19] to analyze the error for the asymmetric technique and get the error estimates between the discrete and analytical solutions. Former papers presented the stability property, 
mentioned that the truncation error is of order $O\left(\Delta t h^{-1}+\right.$ $\Delta t+h)$ for parabolic problems $[20,21]$, or presented the asymmetric technique $[22,23]$ in numerical calculations.

The rest of the paper is organized as follows. In Section 2 the semi-implicit finite difference scheme is presented, and in Section 3 it is proven that the scheme proposed is unconditionally stable for the problem with the order of fractional derivative belonging to $[(\sqrt{17}-1) / 2,2]$. In Section 4 the error estimation is analyzed, and in Section 5 numerical experiments are carried out to verify our theoretical analysis. Finally some concluding remarks are given in the last section.

Throughout the paper, we use $C$ to denote a generic constant, which may have different values in different context.

\section{The Semi-Implicit Finite Difference Method for FDES}

In this section, combining a second-order WSGD operator for the Riemann-Liouville fractional derivatives with the asymmetric discretization technique, the finite difference scheme is constructed for FDEs.

Consider the following one-dimensional space-fractional diffusion equation:

$$
\begin{array}{ll}
\frac{\partial u(x, t)}{\partial t}=c(x, t) \frac{\partial^{\alpha} u(x, t)}{\partial x^{\alpha}}+s(x, t), & \\
x \in(L, R), t \in(0, T], & \\
u(L, t)=0, & t \in(0, T], \\
u(R, t)=b_{R}(t), & \\
u(x, 0)=\phi(x), \quad x \in(L, R) . &
\end{array}
$$

where $\alpha$ is the fractional order and $1<\alpha \leq 2[8,9]$.

\subsection{Second-Order Operator for Riemann-Liouville Fractional} Derivatives. Now from the shifted Grünwald finite difference formula, a second-order WSGD operator is introduced for Riemann-Liouville derivatives. First, some preliminary knowledge involving the Riemann-Liouville derivatives is given [24].

Definition 1. The $\alpha(n-1<\alpha<n)$ order left and right Riemann-Liouville fractional derivatives of the function $u(x)$ on $(a, b)$ are, respectively, defined as follows:

(a) The left Riemann-Liouville fractional derivative:

$$
{ }_{a} D_{x}^{\alpha} u(x)=\frac{1}{\Gamma(n-\alpha)} \frac{d^{n}}{d x^{n}} \int_{a}^{x} \frac{u(\xi)}{(x-\xi)^{\alpha-n+1}} d \xi
$$

(b) The right Riemann-Liouville fractional derivative:

$$
{ }_{x} D_{b}^{\alpha} u(x)=\frac{(-1)^{n}}{\Gamma(n-\alpha)} \frac{d^{n}}{d x^{n}} \int_{x}^{b} \frac{u(\xi)}{(\xi-x)^{\alpha-n+1}} d \xi,
$$

if $\alpha=n,{ }_{a} D_{x}^{\alpha} u(x)=\left(d^{n} / d x^{n}\right) u(x)$ and ${ }_{x} D_{b}^{\alpha} u(x)=(-1)^{n}\left(d^{n} /\right.$ $\left.d x^{n}\right) u(x)$.
The fractional derivative can be approximated by the standard Grüwald formula or the shifted Grüwald formula $[8,9]$ as follows:

$$
\begin{aligned}
& \frac{\partial^{\alpha} u(x, t)}{\partial x^{\alpha}}=\lim _{h \rightarrow 0} \frac{1}{h^{\alpha}} \sum_{k=0}^{M} g_{k} u(x-k h, t), \\
& \frac{\partial^{\alpha} u(x, t)}{\partial x^{\alpha}}=\lim _{h \rightarrow 0} \frac{1}{h^{\alpha}} \sum_{k=0}^{M} g_{k} u(x-(k-1) h, t),
\end{aligned}
$$

where $h=(x-L) / M$ and the coefficients are defined as

$$
g_{k}=\frac{\Gamma(k-\alpha)}{\Gamma(-\alpha) \Gamma(k+1)}=(-1)^{k}\left(\begin{array}{l}
\alpha \\
k
\end{array}\right) \text {. }
$$

Lemma 2 (see $[8,9,24]$ ). The coefficients $\left\{g_{k}\right\}$ satisfy the following properties for $1<\alpha \leqslant 2$,

$$
\begin{aligned}
g_{0} & =1, \quad g_{1}=-\alpha<0,1 \geq g_{2} \geq g_{3} \geq \cdots \geq 0 ; \\
\sum_{k=0}^{m} g_{k} & \leq 0, \quad\left(m \in \mathbb{Z}^{+}\right) ; \\
\sum_{k=0}^{\infty} g_{k} & =0 .
\end{aligned}
$$

Let $\Delta t=T /(2 N)$ and $h=(R-L) / K$ be the time increment and spacial mesh size, respectively. Here $K$ and $N$ are positive integers. For $n=0,1, \ldots,[T / 2 \Delta t]$, and $i=0, \ldots, K$, we denote

$$
\begin{aligned}
t_{n} & =n \Delta t, \\
x_{i} & =L+i h, \\
u_{i}^{n} & =u\left(x_{i}, t_{n}\right), \\
c_{i}^{n} & =c\left(x_{i}, t_{n}\right), \\
s_{i}^{n} & =s\left(x_{i}, t_{n}\right) .
\end{aligned}
$$

Based on the weighted and shifted Grünwald difference (WSGD) operators, the following second-order operator is introduced to approximate the Riemann-Liouville fractional derivative [16] at the grid point $\left(x_{l}, t_{n}\right)$. For $l=1, \ldots, K-1$,

$$
\begin{gathered}
\frac{\partial^{\alpha} u\left(x_{l}, t_{n}\right)}{\partial x^{\alpha}}=\frac{1}{h^{\alpha}}\left\{\left(1-\frac{\alpha}{2}\right) \sum_{k=0}^{l} g_{k} u\left(x_{l}-k h, t_{n}\right)\right. \\
\left.+\frac{\alpha}{2} \sum_{k=0}^{l+1} g_{k} u\left(x_{l}-(k-1) h, t_{n}\right)\right\}+O\left(h^{2}\right) .
\end{gathered}
$$

The simplified form of the approximate formula (11) for the Riemann-Liouville fractional derivative is

$$
\begin{aligned}
\frac{\partial^{\alpha} u\left(x_{l}, t_{n}\right)}{\partial x^{\alpha}}= & \frac{1}{h^{\alpha}} \sum_{k=0}^{l+1} w_{k} u\left(x_{l}-(k-1) h, t_{n}\right) \\
& +O\left(h^{2}\right),
\end{aligned}
$$


where

$$
\begin{aligned}
& w_{0}=\frac{\alpha}{2}, \\
& w_{k}=\frac{\alpha}{2} g_{k}+\left(1-\frac{\alpha}{2}\right) g_{k-1}, \quad k \geqslant 1 .
\end{aligned}
$$

From Lemma 2 and some straightforward calculations, we obtain the following properties of the coefficient $\left\{w_{k}\right\}$ in (12) [16].

Lemma 3 (see [16]). The coefficients $\left\{w_{k}\right\}$ satisfy the following properties for $\alpha \in[(\sqrt{17}-1) / 2,2]$,

$$
\begin{aligned}
1 & \geqslant w_{0} \geqslant w_{2} \geqslant w_{3} \geqslant w_{4} \geqslant \cdots \geqslant 0, \quad w_{1}<0 ; \\
\sum_{k=0}^{M} w_{k} & \leqslant 0, \quad M \in \mathbb{Z}^{+} ; \\
\sum_{k=0}^{\infty} w_{k} & =0 .
\end{aligned}
$$

Combining (12) with the implicit Euler discretization, the finite difference scheme for solving equations (1)-(3) is introduced.

$$
\frac{v_{l}^{n+1}-v_{l}^{n}}{\Delta t}=\frac{c_{l}^{n+1}}{h^{\alpha}} \sum_{k=0}^{l+1} w_{k} v_{l-k+1}^{n+1}+s_{l}^{n+1}+O\left(\Delta t+h^{2}\right),
$$

where $l=1, \ldots, K-1$.

Now, the following semi-implicit finite difference scheme is constructed using the asymmetric technique $[18,19]$.

For $1 \leq l \leq K-1$ and $0 \leq n \leq N=T / 2 \Delta t$, one goal is to find $\left\{v_{l}^{2 n+2}\right\}$ such that

$$
\begin{aligned}
& \text { (a) } \frac{v_{l}^{2 n+1}-v_{l}^{2 n}}{\Delta t}=\frac{c_{l}^{2 n+1}}{h^{\alpha}}\left[\sum_{k=2}^{l+1} w_{k} v_{l-k+1}^{2 n+1}\right. \\
& \left.+\left(w_{1}+\frac{\alpha}{2}\right) v_{l}^{2 n+1}-\frac{\alpha}{2} v_{l}^{2 n}+\frac{\alpha}{2} v_{l+1}^{2 n}\right]+s_{l}^{2 n+1}, \\
& \text { (b) } \frac{v_{l}^{2 n+2}-v_{l}^{2 n+1}}{\Delta t}=\frac{c_{l}^{2 n+2}}{h^{\alpha}}\left[\sum_{k=2}^{l+1} w_{k} v_{l-k+1}^{2 n+1}\right. \\
& \left.+\left(w_{1}+\frac{\alpha}{2}\right) v_{l}^{2 n+1}-\frac{\alpha}{2} v_{l}^{2 n+2}+\frac{\alpha}{2} v_{l+1}^{2 n+2}\right]+s_{l}^{2 n+2} .
\end{aligned}
$$

The initial and boundary conditions are as follows:

$$
\begin{aligned}
v_{l}^{0} & =\phi\left(x_{l}\right), \quad l=0,1, \ldots, K ; \\
v_{0}^{n} & =0, \\
v_{K}^{n} & =b_{R}\left(R, t^{n}\right) .
\end{aligned}
$$

The scheme is an implicit scheme formally. In order to solve it explicitly, we calculate the unknowns according to the following nodal-point sequences. Here different nodal-point stencils are given at levels $2 n+1$ and $2 n+2$ (see Figures 1-2).

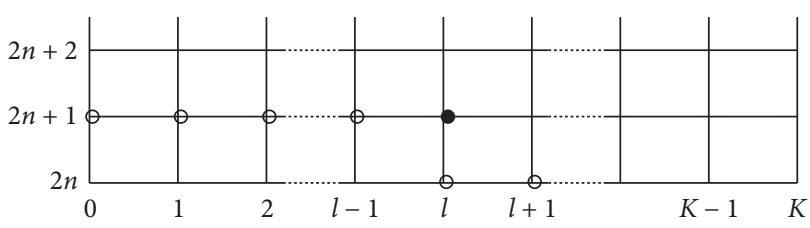

Figure 1: The finite difference stencil for point $(l, 2 n+1)$.

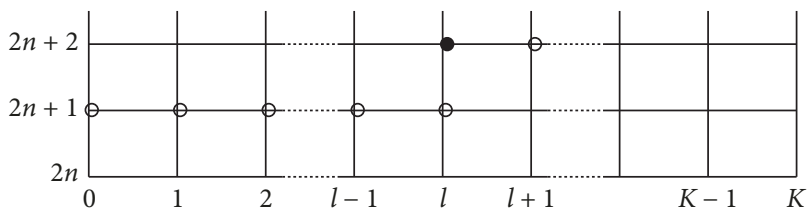

FIgURE 2: The finite difference stencil for point $(l, 2 n+2)$.

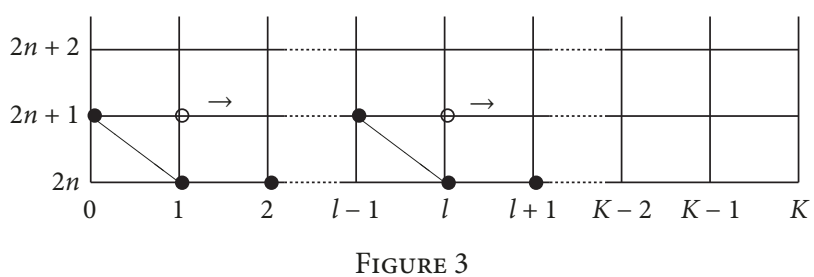

The specific calculation processes are as follows. Let

$$
p_{l}^{n}=\frac{c_{l}^{n} \Delta t}{h^{\alpha}} .
$$

From (16),

$$
\begin{aligned}
(1- & \left.p_{l}^{2 n+1}\left(w_{1}+\frac{\alpha}{2}\right)\right) v_{l}^{2 n+1} \\
= & \sum_{k=2}^{l+1} p_{l}^{2 n+1} w_{k} v_{l-k+1}^{2 n+1}+\left(1-p_{l}^{2 n+1} \frac{\alpha}{2}\right) v_{l}^{2 n} \\
& +p_{l}^{2 n+1} \frac{\alpha}{2} v_{l+1}^{2 n}+\Delta t s_{l}^{2 n+1},
\end{aligned}
$$

and

$$
\begin{aligned}
(1+ & \left.p_{l}^{2 n+2} \frac{\alpha}{2}\right) v_{l}^{2 n+2} \\
= & \sum_{k=2}^{l+1} p_{l}^{2 n+2} w_{k} v_{l-k+1}^{2 n+1}+\left(1+p_{l}^{2 n+2}\left(w_{1}+\frac{\alpha}{2}\right)\right) v_{l}^{2 n+1} \\
& +p_{l}^{2 n+2} \frac{\alpha}{2} v_{l+1}^{2 n+2}+\Delta t s_{l}^{2 n+2} .
\end{aligned}
$$

(1) Solve (19) according to the following sequence from timestep $t_{2 n}$ to time-step $t_{2 n+1}:(2 n+1,1) \longrightarrow(2 n+1,2) \longrightarrow \cdots \longrightarrow$ $(2 n+1, l-1) \longrightarrow(2 n+1, l) \longrightarrow \cdots($ cf. Figure 3$)$.

Once we find $v_{l-k+1}^{2 n+1}$ for $k \leq l-1$, combining with $v_{l}^{2 n}$ and $v_{l+1}^{2 n}, v_{l}^{2 n+1}$ can be obtained explicitly.

(2) Solve (20) according to the following sequence from time $t^{2 n+1}$ to time $t^{2 n+2}:(2 n+2, K-1) \longrightarrow(2 n+2, K-2) \longrightarrow$ $\cdots \longrightarrow(2 n+2, l) \longrightarrow(2 n+2, l-1) \longrightarrow \cdots($ cf. Figure 4$)$.

Similarly, $v_{l}^{2 n+2}$ can be derived explicitly. 


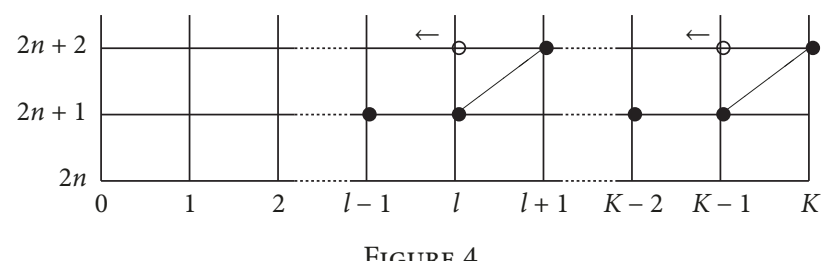

FIgURe 4

In conclusion, the discrete solutions of (19) and (20) can be obtained explicitly one by one instead of solving the linear algebraic system.

\section{Stability of the Semi-Implicit Finite Difference Scheme}

In this section, it is proven that the presented scheme (16) is uniformly stable as $\alpha \in[(\sqrt{17}-1) / 2,2]$.

Suppose the coefficient of the diffusion term $c(x, t)=c$ is a constant. Then $p_{i}^{n}=p$ is a constant.

From (19) and (20),

$$
\begin{gathered}
\sum_{k=2}^{l+1}-p w_{k} v_{l-k+1}^{2 n+1}+\left(1-p\left(w_{1}+\frac{\alpha}{2}\right)\right) v_{l}^{2 n+1} \\
=\left(1-\frac{\alpha}{2} p\right) v_{l}^{2 n}+\frac{\alpha}{2} p v_{l+1}^{2 n}+\Delta t s_{l}^{2 n+1}, \\
\left(1+\frac{\alpha}{2} p\right) v_{l}^{2 n+2}-\frac{\alpha}{2} p v_{l+1}^{2 n+2} \\
=\sum_{k=2}^{l+1} p w_{k} v_{l-k+1}^{2 n+1}+\left(1+p\left(w_{1}+\frac{\alpha}{2}\right)\right) v_{l}^{2 n+1} \\
\quad+\Delta t s_{l}^{2 n+2} .
\end{gathered}
$$

Equation (21) can be written as the following linear system:

$$
\begin{aligned}
& (I+A) V^{2 n+1}=(I-B) V^{2 n}+\Delta t S^{2 n+1}, \\
& (I+B) V^{2 n+2}=(I-A) V^{2 n+1}+\Delta t S^{2 n+2},
\end{aligned}
$$

where for nonnegative integer $m$,

$$
\begin{aligned}
V^{m} & =\left(v_{1}^{m}, v_{2}^{m}, \ldots, v_{K-1}^{m}\right)^{T}, \\
S^{m} & =\left(s_{1}^{m}, s_{2}^{m}, \ldots, s_{K-1}^{m}\right)^{T} .
\end{aligned}
$$

The matrix entries $A_{i j}$ and $B_{i j}$ for $i=1, \ldots, K-1$ and $j=$ $1, \ldots, K-1$ are defined by

$$
A_{i j}= \begin{cases}0 & j \geq i+1 \\ -p\left(w_{1}+\frac{\alpha}{2}\right) & j=i \\ -p w_{i-j+1} & j \leq i-1,\end{cases}
$$

$$
B_{i j}= \begin{cases}0 & j \geq i+2 \\ -\frac{\alpha}{2} p & j=i+1 \\ \frac{\alpha}{2} p & j=i \\ 0 & j \leq i-1 .\end{cases}
$$

Using the Fourier method, the stability of the presented scheme (16) with the initial value is proven. In general, we suppose $s=0$ and $b_{R}=0$, then (21) becomes

$$
\begin{aligned}
& \sum_{k=2}^{l+1}-p w_{k} v_{l-k+1}^{2 n+1}+\left(1-p\left(w_{1}+\frac{\alpha}{2}\right)\right) v_{l}^{2 n+1} \\
& =\left(1-\frac{\alpha}{2} p\right) v_{l}^{2 n}+\frac{\alpha}{2} p v_{l+1}^{2 n}, \\
& \left(1+\frac{\alpha}{2} p\right) v_{l}^{2 n+2}-\frac{\alpha}{2} p v_{l+1}^{2 n+2} \\
& =\sum_{k=2}^{l+1} p w_{k} v_{l-k+1}^{2 n+1}+\left(1+p\left(w_{1}+\frac{\alpha}{2}\right)\right) v_{l}^{2 n+1} .
\end{aligned}
$$

Let

$$
v_{l}^{2 n+1}=Z^{2 n+1} e^{i \beta l h}
$$

where $\beta$ is a nonnegative integer.

So

$$
\begin{aligned}
& \left\{\sum_{k=2}^{l+1}-p w_{k} e^{-i \beta(k-1) h}+1-p\left(w_{1}+\frac{\alpha}{2}\right)\right\} Z^{2 n+1} e^{i \beta l h} \\
& \quad=\left(1-\frac{\alpha}{2} p+\frac{\alpha}{2} p e^{i \beta h}\right) Z^{2 n} e^{i \beta l h}
\end{aligned}
$$

and

$$
\begin{aligned}
& \left(1+\frac{\alpha}{2} p-\frac{\alpha}{2} p e^{i \beta h}\right) Z^{2 n+2} e^{i \beta l h} \\
& =\left\{\sum_{k=2}^{l+1} p w_{k} e^{-i \beta(k-1) h}+1+p\left(w_{1}+\frac{\alpha}{2}\right)\right\} Z^{2 n+1} e^{i \beta l h} .
\end{aligned}
$$

Then

$$
Z^{2 n+2}=G^{2 n+2} Z^{2 n}
$$

where

$$
\begin{aligned}
& G^{2 n+2}=\frac{\left(1-(\alpha / 2) p+(\alpha / 2) p e^{i \beta h}\right)}{\left(1+(\alpha / 2) p-(\alpha / 2) p e^{i \beta h}\right)} \\
& \cdot \frac{\sum_{k=2}^{l+1} p w_{k} e^{-i \beta(k-1) h}+1+p\left(w_{1}+\alpha / 2\right)}{\sum_{k=2}^{l+1}-p w_{k} e^{-i \beta(k-1) h}+1-p\left(w_{1}+\alpha / 2\right)} \\
& =\frac{\left(1-(\alpha / 2) p+(\alpha / 2) p e^{i \beta h}\right)}{\left(1+(\alpha / 2) p-(\alpha / 2) p e^{i \beta h}\right)} \\
& \cdot \frac{\sum_{k=1}^{l} p w_{k+1} e^{-i \beta k h}+1+p\left(w_{1}+\alpha / 2\right)}{\sum_{k=1}^{l}-p w_{k+1} e^{-i \beta k h}+1-p\left(w_{1}+\alpha / 2\right)} \equiv G_{1} G_{2} .
\end{aligned}
$$


Here

$$
\begin{aligned}
& G_{1}=\frac{a_{1}+i(\alpha p / 2) \sin \beta h}{a_{2}-i(\alpha p / 2) \sin \beta h}, \\
& G_{2}=\frac{b_{1}-i c}{b_{2}+i c} .
\end{aligned}
$$

According to Lemma 3,

$$
\begin{aligned}
& a_{1}=1-\frac{\alpha}{2} p+\frac{\alpha}{2} p \cos (\beta h), \\
& a_{2}=1+\frac{\alpha}{2} p-\frac{\alpha}{2} p \cos (\beta h), \\
& b_{1}=1+\frac{p\left(2-\alpha^{2}\right)}{2}+\sum_{k=1}^{l} p w_{k+1} \cos (\beta k h), \\
& b_{2}=1-\frac{p\left(2-\alpha^{2}\right)}{2}-\sum_{k=1}^{l} p w_{k+1} \cos (\beta k h), \\
& c=\sum_{k=1}^{l} p w_{k+1} \sin (\beta k h) .
\end{aligned}
$$

Lemma 4. If $s=0$ and $b_{R}=0$ in (21) with $\alpha \in[(\sqrt{17}-1) / 2,2]$,

$$
\left\|V^{2 n+2}\right\| \leq\left\|V^{2 n}\right\| \text {. }
$$

Proof. First, inequality $\left|G_{2}\right| \leq 1$ is proved. It is obvious that $\left|\left(b_{1}-i c\right) /\left(b_{2}+i c\right)\right|=\left|\left(b_{1}-i c\right) /\left(b_{2}-i c\right)\right|$, so

$$
\begin{aligned}
\left|G_{2}\right|^{2}-1 & =\frac{b_{1}^{2}-b_{2}^{2}}{b_{2}^{2}+c^{2}} \\
& =\frac{4 p\left(\left(2-\alpha^{2}\right) / 2+\sum_{k=1}^{l} w_{k+1} \cos (\beta k h)\right)}{b_{2}^{2}+c^{2}} .
\end{aligned}
$$

From Lemmas 2 and 3, we have

$$
\begin{aligned}
\left|\sum_{k=1}^{l} w_{k+1} \cos (\beta k h)\right| & \leq \sum_{k=1}^{l} w_{k+1} \\
& =\sum_{k=1}^{l}\left[\frac{\alpha}{2} g_{k+1}+\left(1-\frac{\alpha}{2}\right) g_{k}\right] \\
& =\frac{\alpha}{2} \sum_{k=1}^{l} g_{k+1}+\left(1-\frac{\alpha}{2}\right) \sum_{k=1}^{l} g_{k} \\
& \leq \frac{\alpha}{2}(\alpha-1)-\left(1-\frac{\alpha}{2}\right) \\
& =\frac{\alpha^{2}}{2}-1 .
\end{aligned}
$$

So $\left|G_{2}\right|^{2} \leq 1$. Furthermore

$$
\left|G_{2}\right| \leq 1 \text {. }
$$

In the same way, $\left|G_{1}\right| \leq 1$ is obtained. On combining with (37) and (31), we have

$$
\left|G^{2 n+2}\right| \leq 1 .
$$

This completes the proof.
Define

$$
\begin{aligned}
& V^{2 n+1}=\widehat{V}^{2 n+1}+\widetilde{V}^{2 n+1}, \\
& V^{2 n+2}=\widehat{V}^{2 n+2}+\widetilde{V}^{2 n+2} .
\end{aligned}
$$

Then the linear system (22) can be written as follows:

$$
\begin{aligned}
& (I+A) \widehat{V}^{2 n+1}=(I-B) V^{2 n}, \\
& (I+A) \widetilde{V}^{2 n+1}=\Delta t S^{2 n+1}, \\
& (I+B) \widehat{V}^{2 n+2}=(I-A) \widehat{V}^{2 n+1}, \\
& (I+B) \widetilde{V}^{2 n+2}=(I-A) \widetilde{V}^{2 n+1}+\Delta t S^{2 n+2} .
\end{aligned}
$$

Through simple computations,

$$
\begin{aligned}
& (I+A) \widehat{V}^{2 n+1}=(I-B) V^{2 n}, \\
& (I+B) \widehat{V}^{2 n+2}=(I-A) \widehat{V}^{2 n+1} .
\end{aligned}
$$

Then we have

$$
(I+B) \widetilde{V}^{2 n+2}=(I-A)(I+A)^{-1} \Delta t S^{2 n+1}+\Delta t S^{2 n+2} .
$$

Lemma 5. The eigenvalues of matrices $A$ and $B$ are nonnegative.

Proof. Let $\lambda$ be an eigenvalue of matrix $A$. According to Gerschgorim theorem [25], for every eigenvalue $\lambda$, it holds

$$
A_{l l}-R \leq \lambda \leq A_{l l}+R,
$$

where $R$ is the radius and

$$
R=\sum_{j=1, j \neq l}^{K-1} A_{l j}=\sum_{j=1}^{l-1} p w_{l-j+1}=p \sum_{j=2}^{l} w_{j} .
$$

By inequality (36), it is obvious that

$$
\begin{aligned}
A_{l l}-R_{l} & =-p\left(w_{1}+\frac{\alpha}{2}\right)-p \sum_{j=2}^{l} w_{j} \\
& =p\left(\frac{\alpha^{2}}{2}-1\right)-p \sum_{j=2}^{l} w_{j} \geqslant 0 .
\end{aligned}
$$

From (43), we derive that $\lambda \geqslant 0$. The eigenvalues of matrix $B$ which are nonnegative can be proven in the same way.

The above two lemmas are sewn together to yield the following theorem that guarantees the stability of the presented scheme (16).

Theorem 6. Suppose $c(x, t)=c$ is a constant function. If $b_{R}=$ 0 , the scheme is uniformly stable about the initial value and right-hand side term with $\alpha \in[(\sqrt{17}-1) / 2,2]$ and

$$
\left\|V^{2 n+2}\right\| \leq\left\|V^{0}\right\|+\sum_{m=1}^{2 n+2} \Delta t\left\|S^{m}\right\| .
$$


Proof. Using Lemma 5, we obtain

$$
\begin{aligned}
\|I+B\| & \geq \max \lambda_{I+B} \geq 1, \\
\left\|(I+B)^{-1}\right\| & =\|I+B\|^{-1} \leq 1,
\end{aligned}
$$

and

$$
\left\|(I-A)(I+A)^{-1}\right\| \leq 1 .
$$

By (42),

$$
\begin{aligned}
\left\|\widetilde{V}^{2 n+2}\right\| \leq & \left\|(I+B)^{-1}\right\|\left\|(I-A)(I+A)^{-1}\right\| \Delta t\left\|S^{2 n+1}\right\| \\
& +\Delta t\left\|S^{2 n+2}\right\| \leq \Delta t\left\|S^{2 n+1}\right\|+\Delta t\left\|S^{2 n+2}\right\| .
\end{aligned}
$$

From (41) and Lemma 4,

$$
\left\|\widehat{V}^{2 n+2}\right\| \leq\left\|V^{2 n}\right\|
$$

Combining (49), (50) with (39),

$$
\begin{aligned}
\left\|V^{2 n+2}\right\|= & \left\|\widehat{V}^{2 n+2}+\widetilde{V}^{2 n+2}\right\| \\
\leq & \left\|V^{2 n}\right\|+\Delta t\left\|S^{2 n+1}\right\|+\Delta t\left\|S^{2 n+2}\right\| \\
\leq & \left(\left\|V^{2 n-2}\right\|+\Delta t\left\|S^{2 n-1}\right\|+\Delta t\left\|S^{2 n}\right\|\right) \\
& +\Delta t\left\|S^{2 n+1}\right\|+\Delta t\left\|S^{2 n+2}\right\| \ldots \\
\leq & \left\|V^{0}\right\|+\sum_{m=1}^{2 n+2} \Delta t\left\|S^{m}\right\| .
\end{aligned}
$$

This completes the proof.

\section{Error Estimate for the Finite Difference Scheme}

In this section, the error estimate is considered for the presented scheme (16). Suppose $c(x, t)=c$ is a constant. have

Let $u_{l}^{n}$ be the exact solution of (1) (3) at $\left(x_{l}, t_{n}\right)$, then we

$$
\begin{aligned}
& \frac{u_{l}^{2 n+1}-u_{l}^{2 n}}{\Delta t}-s_{l}^{2 n+1}-\frac{c}{h^{\alpha}}\left\{\sum_{k=2}^{l+1} w_{k} u_{l-k+1}^{2 n+1}\right. \\
& \left.+\left(w_{1}+\frac{\alpha}{2}\right) u_{l}^{2 n+1}-\frac{\alpha}{2} u_{l}^{2 n}+\frac{\alpha}{2} u_{l+1}^{2 n}\right\} \\
& =\frac{u_{l}^{2 n+1}-u_{l}^{2 n}}{\Delta t}-\left(\frac{\partial u}{\partial t}\right)_{l}^{2 n+1}-\frac{c}{h^{\alpha}} \sum_{k=0}^{l+1} w_{k} u_{l-k+1}^{2 n+1} \\
& +\left(c \frac{\partial^{\alpha} u}{\partial x^{\alpha}}\right)_{l}^{2 n+1}+\frac{c}{h^{\alpha}} \frac{\alpha}{2}\left(u_{l+1}^{2 n+1}-u_{l}^{2 n+1}\right. \\
& \left.-\left(u_{l+1}^{2 n}-u_{l}^{2 n}\right)\right) .
\end{aligned}
$$

Using Taylor expansion,

$$
\begin{aligned}
\frac{1}{h}\left(u_{l+1}^{2 n+1}-u_{l}^{2 n+1}-\left(u_{l+1}^{2 n}-u_{l}^{2 n}\right)\right) \\
=\left(\frac{\partial u}{\partial x}\right)_{l}^{2 n+1}-\left(\frac{\partial u}{\partial x}\right)_{l}^{2 n} \\
+\frac{h}{2}\left[\left(\frac{\partial^{2} u}{\partial x^{2}}\right)_{l}^{2 n+1}-\left(\frac{\partial^{2} u}{\partial x^{2}}\right)_{l}^{2 n}\right] \\
+\frac{h^{2}}{6}\left[\left(\frac{\partial^{3} u}{\partial x^{3}}\right)_{l}^{2 n+1}-\left(\frac{\partial^{3} u}{\partial x^{3}}\right)_{l}^{2 n}\right]+O\left(h^{3}\right) \\
=\Delta t\left(\frac{\partial^{2} u}{\partial x \partial t}\right)_{l}^{2 n+1}+O\left(\Delta t^{2}+\Delta t h+\Delta t h^{2}+h^{3}\right)
\end{aligned}
$$

at time-step $t^{2 n+1}$.

So, (52) can be written as follows:

$$
\begin{aligned}
& \frac{u_{l}^{2 n+1}-u_{l}^{2 n}}{\Delta t}-s_{l}^{2 n+1}-\frac{c}{h^{\alpha}}\left\{\sum_{k=2}^{l+1} w_{k} u_{l-k+1}^{2 n+1}\right. \\
& \left.+\left(w_{1}+\frac{\alpha}{2}\right) u_{l}^{2 n+1}-\frac{\alpha}{2} u_{l}^{2 n}+\frac{\alpha}{2} u_{l+1}^{2 n}\right\}=O(\Delta t \\
& \left.+h^{2}\right)+\frac{c \Delta t}{h^{\alpha-1}} \frac{\alpha}{2}\left(\frac{\partial^{2} u}{\partial x \partial t}\right)_{l}^{2 n+1}+O\left(\Delta t^{2} h^{1-\alpha}\right. \\
& \left.+\Delta t h^{2-\alpha}+\Delta t h^{3-\alpha}+h^{4-\alpha}\right) .
\end{aligned}
$$

Since $1<\alpha<2$,

$$
\begin{aligned}
& \frac{u_{l}^{2 n+1}-u_{l}^{2 n}}{\Delta t}-s_{l}^{2 n+1}-\frac{c}{h^{\alpha}}\left\{\sum_{k=2}^{l+1} w_{k} u_{l-k+1}^{2 n+1}\right. \\
& \left.+\left(w_{1}+\frac{\alpha}{2}\right) u_{l}^{2 n+1}-\frac{\alpha}{2} u_{l}^{2 n}+\frac{\alpha}{2} u_{l+1}^{2 n}\right\}=\frac{c \Delta t}{h^{\alpha-1}} \\
& \cdot \frac{\alpha}{2}\left(\frac{\partial^{2} u}{\partial x \partial t}\right)_{l}^{2 n+1}+O\left(\Delta t^{2} h^{1-\alpha}+\Delta t+h^{2}\right) .
\end{aligned}
$$

In the same way,

$$
\begin{aligned}
& \frac{u_{l}^{2 n+2}-u_{l}^{2 n+1}}{\Delta t}-s_{l}^{2 n+2}-\frac{c}{h^{\alpha}}\left\{\sum_{k=2}^{l+1} w_{k} u_{l-k+1}^{2 n+1}\right. \\
& \left.+\left(w_{1}+\frac{\alpha}{2}\right) u_{l}^{2 n+1}-\frac{\alpha}{2} u_{l}^{2 n+2}+\frac{\alpha}{2} u_{l+1}^{2 n+2}\right\}=-\frac{c \Delta t}{h^{\alpha-1}} \\
& \cdot \frac{\alpha}{2}\left(\frac{\partial^{2} u}{\partial x \partial t}\right)_{l}^{2 n+1}+O\left(\Delta t^{2} h^{1-\alpha}+\Delta t+h^{2}\right)
\end{aligned}
$$

at time-step $t^{2 n+2}$.

Let

$$
U^{m}=\left(u_{1}^{m}, u_{2}^{m}, \ldots, u_{K-1}^{m}\right) .
$$


Theorem 7. Let $u\left(x_{l}, t_{n}\right)$ be the exact solution of (1) (3) and $v_{l}^{n}$ be the solution of (16). Suppose $c(x, t)$ is a constant function. When $h$ and $\Delta t$ are sufficiently small, there exists a positive constant $C$ independent of $\Delta t$ and $h$ such that for $m \leq T / \Delta t$,

$$
\left\|V^{m}-U^{m}\right\| \leq C\left(\Delta t^{2} h^{-2(\alpha-1)}+\Delta t+h^{2}\right) .
$$

Proof. We define $e_{l}^{2 n+1}$ as follows at the odd time-step $t^{2 n+1}$

$$
\begin{array}{ll}
e_{0}^{2 n+1}=v_{0}^{2 n+1}-u_{0}^{2 n+1}= & 0, \\
e_{l}^{2 n+1}=v_{l}^{2 n+1}-u_{l}^{2 n+1}+\frac{c \Delta t^{2}}{h^{\alpha-1}} \frac{\alpha}{2}\left(\frac{\partial^{2} u}{\partial x \partial t}\right)_{l}^{2 n+1}, & 1 \leq l \leq K-1, \\
e_{K}^{2 n+1}=v_{K}^{2 n+1}-u_{K}^{2 n+1}=0 . &
\end{array}
$$

Similarly, we define $e_{l}^{2 n+2}$ the even time-step $t^{2 n+2}$

$$
\begin{aligned}
& e_{0}^{2 n+2}=v_{0}^{2 n+2}-u_{0}^{2 n+2}=0, \\
& e_{l}^{2 n+2}=v_{l}^{2 n+2}-u_{l}^{2 n+2}, \quad 1 \leq l \leq K-1, \\
& e_{K}^{2 n+2}=v_{K}^{2 n+2}-u_{K}^{2 n+2}=0 .
\end{aligned}
$$

According to (56), (55), and (16),

$$
\begin{aligned}
& \frac{e_{l}^{2 n+1}-e_{l}^{2 n}}{\Delta t}=\frac{c}{h^{\alpha}}\left\{\sum_{k=2}^{l+1} w_{k} e_{l-k+1}^{2 n+1}+\left(w_{1}+\frac{\alpha}{2}\right) e_{l}^{2 n+1}\right. \\
& \left.-\frac{\alpha}{2} e_{l}^{2 n}+\frac{\alpha}{2} e_{l+1}^{2 n}\right\}-\frac{c \Delta t^{2}}{h^{2 \alpha-1}} \frac{\alpha}{2} \\
& \quad \sum_{k=2}^{l+1} w_{k}\left(\left(c \frac{\partial^{2} u}{\partial x \partial t}\right)_{l-k+1}^{2 n+1}-\left(c \frac{\partial^{2} u}{\partial x \partial t}\right)_{l}^{2 n+1}\right) \\
& +O\left(\Delta t^{2} h^{1-\alpha}+\Delta t+h^{2}\right), \\
& \frac{e_{l}^{2 n+2}-e_{l}^{2 n+1}}{\Delta t}=\frac{c}{h^{\alpha}}\left\{\sum_{k=2}^{l+1} w_{k} e_{l-k+1}^{2 n+1}+\left(w_{1}+\frac{\alpha}{2}\right) e_{l}^{2 n+1}\right. \\
& \left.\quad-\frac{\alpha}{2} e_{l}^{2 n+2}+\frac{\alpha}{2} e_{l+1}^{2 n+2}\right\}_{l}-\frac{c \Delta t^{2}}{h^{2 \alpha-1}} \frac{\alpha}{2} \\
& +\sum_{k=2}^{l+1} w_{k}\left(\left(c \frac{\partial^{2} u}{\partial x \partial t}\right)_{l-k+1}^{2 n+1}-\left(c \frac{\partial^{2} u}{\partial x \partial t}\right)_{l}^{2 n+1}\right) \\
& +O\left(\Delta t^{2} h^{1-\alpha}+\Delta t+h^{2}\right) .
\end{aligned}
$$

Using Taylor expansion,

$$
\left(c \frac{\partial^{2} u}{\partial x \partial t}\right)_{l-k+1}^{2 n+1}-\left(c \frac{\partial^{2} u}{\partial x \partial t}\right)_{l}^{2 n+1}=O((k-1) h) .
$$

Through the estimates for the $g_{k}$ in [9], there exist two constants $K_{1}$ and $K_{2}$ such that

$$
\frac{K_{1}}{k^{\alpha+1}} \leq g_{k} \leq \frac{K_{2}}{k^{\alpha+1}}
$$

Combining (12) with (63), we know

$$
\frac{C_{1}}{k^{\alpha+1}} \leq w_{k} \leq \frac{C_{2}}{k^{\alpha+1}}
$$

Here $C_{1}$ and $C_{2}$ are constant.

Using (62) and (64), we have

$$
\begin{aligned}
& \left|w_{k}\left(\left(c \frac{\partial^{2} u}{\partial x \partial t}\right)_{l-k+1}^{2 n+1}-\left(c \frac{\partial^{2} u}{\partial x \partial t}\right)_{l}^{2 n+1}\right)\right| \\
& \leq \frac{1}{k^{\alpha+1}} C(k-1) h \leq \frac{C_{3}}{k^{\alpha}} h .
\end{aligned}
$$

Here $C$ and $C_{3}$ are constant.

Since $1<\alpha<2$, there exists a positive constant $K$ independent of $h$ such that

$$
\begin{aligned}
& \left|\sum_{k=2}^{l+1} w_{k}\left(\left(c \frac{\partial^{2} u}{\partial x \partial t}\right)_{l-k+1}^{2 n+1}-\left(c \frac{\partial^{2} u}{\partial x \partial t}\right)_{l}^{2 n+1}\right)\right| \leq \sum_{k=2}^{l+1} \frac{C_{3}}{k^{\alpha}} h \\
& \quad \leq K h .
\end{aligned}
$$

So (61) can be written as follows:

$$
\begin{gathered}
\frac{e_{l}^{2 n+1}-e_{l}^{2 n}}{\Delta t}=\frac{c}{h^{\alpha}}\left\{\sum_{k=2}^{l+1} w_{k} e_{l-k+1}^{2 n+1}+\left(w_{1}+\frac{\alpha}{2}\right) e_{l}^{2 n+1}\right. \\
\left.-\frac{\alpha}{2} e_{l}^{2 n}+\frac{\alpha}{2} e_{l+1}^{2 n}\right\}+O\left(\Delta t^{2} h^{-2(\alpha-1)}+\Delta t+h^{2}\right), \\
\frac{e_{l}^{2 n+2}-e_{l}^{2 n+1}}{\Delta t}=\frac{c}{h^{\alpha}}\left\{\sum_{k=2}^{l+1} w_{k} e_{l-k+1}^{2 n+1}+\left(w_{1}+\frac{\alpha}{2}\right) e_{l}^{2 n+1}\right. \\
\left.-\frac{\alpha}{2} e_{l}^{2 n+2}+\frac{\alpha}{2} e_{l+1}^{2 n+2}\right\}+O\left(\Delta t^{2} h^{-2(\alpha-1)}+\Delta t+h^{2}\right) .
\end{gathered}
$$

It is obvious that

$$
e_{l}^{0}=0, \quad 0 \leq l \leq K .
$$

Set

$$
E^{m}=\left(e_{1}^{m}, e_{2}^{m}, \ldots, e_{K-1}^{m}\right) .
$$

Using Theorem 6,

$$
\left\|E^{m}\right\| \leq C\left(\Delta t^{2} h^{-2(\alpha-1)}+\Delta t+h^{2}\right) .
$$

Remark 8. From (55) the truncation error of the scheme (16) is $\left(O(\Delta t h)^{-(\propto-1)}+\Delta t+h^{2}\right)$. Here it is proven that the error between the discrete and the analytical solutions is bounded by $\left(C\left((\Delta t)^{2} h\right)^{-2(\propto-1)}+\Delta t+h^{2}\right)$ by using the asymmetric discretisation technique.

\section{Numerical Experiments}

In this section, numerical examples of one-dimensional cases are presented to show the effectiveness and convergence orders of the scheme (16). 
TABLE 1: Error and convergence rates for Example 1.

\begin{tabular}{lccccc}
\hline & \multicolumn{2}{c}{$\alpha=1.6, \Delta t=4 h^{1.6}$} & & \\
$(1 / h, 1 / \Delta t)$ & $\|e\|_{l^{\infty}}$ & Rate & Rate & CPU(s) \\
\hline$(10,10)$ & $8.1450 \times 10^{-2}$ & - & $2.5887 \times 10^{-2}$ & - & 0.001 \\
\hline$(20,32)$ & $2.3726 \times 10^{-2}$ & 1.78 & $5.3215 \times 10^{-3}$ & 2.28 & 0.001 \\
\hline$(40,92)$ & $8.0849 \times 10^{-3}$ & 1.55 & $1.2802 \times 10^{-3}$ & 2.06 & 0.003 \\
\hline$(80,278)$ & $2.6561 \times 10^{-3}$ & 1.61 & $3.0010 \times 10^{-4}$ & 2.09 & 0.026 \\
\hline$(160,840)$ & $8.7693 \times 10^{-4}$ & 1.60 & $7.7019 \times 10^{-5}$ & 1.96 & 0.12 \\
\hline$(320,2548)$ & $2.8887 \times 10^{-4}$ & 1.60 & $2.3741 \times 10^{-5}$ & 1.70 & \\
\hline
\end{tabular}

TABLE 2: Error and convergence rates for Example 1.

\begin{tabular}{|c|c|c|c|c|c|}
\hline \multicolumn{6}{|c|}{$\alpha=1.8, \Delta t=5 h^{1.8}$} \\
\hline$(1 / h, 1 / \Delta t)$ & $\|e\|_{l^{\infty}}$ & Rate & $\|e\|_{l^{2}}$ & Rate & $\mathrm{CPU}(\mathrm{s})$ \\
\hline$(10,14)$ & $5.6493 \times 10^{-2}$ & - & $2.3870 \times 10^{-2}$ & - & 0.001 \\
\hline$(20,44)$ & $1.7108 \times 10^{-2}$ & 1.72 & $6.6279 \times 10^{-3}$ & 1.85 & 0.002 \\
\hline$(40,154)$ & $4.8088 \times 10^{-3}$ & 1.83 & $1.5575 \times 10^{-3}$ & 2.09 & 0.007 \\
\hline$(80,534)$ & $1.3804 \times 10^{-3}$ & 1.82 & $3.7624 \times 10^{-4}$ & 2.05 & 0.034 \\
\hline$(160,1856)$ & $3.9664 \times 10^{-4}$ & 1.80 & $9.0186 \times 10^{-5}$ & 2.06 & 0.389 \\
\hline$(320,6462)$ & $1.1384 \times 10^{-4}$ & 1.80 & $2.1685 \times 10^{-5}$ & 2.06 & 2.861 \\
\hline
\end{tabular}

TABLE 3: Error and convergence rates for Example 1.

\begin{tabular}{|c|c|c|c|c|c|}
\hline \multicolumn{6}{|c|}{$\alpha=1.6, \Delta t=5 h^{2}$} \\
\hline$(1 / h, 1 / \Delta t)$ & $\|e\|_{l \infty}$ & Rate & $\|e\|_{l^{2}}$ & Rate & $\mathrm{CPU}(\mathrm{s})$ \\
\hline$(10,20)$ & $3.8690 \times 10^{-2}$ & - & $1.2379 \times 10^{-2}$ & - & 0.001 \\
\hline$(20,80)$ & $9.3129 \times 10^{-3}$ & 2.05 & $2.1854 \times 10^{-3}$ & 2.50 & 0.003 \\
\hline$(40,320)$ & $2.3064 \times 10^{-3}$ & 2.01 & $4.2095 \times 10^{-4}$ & 2.38 & 0.017 \\
\hline$(80,1280)$ & $5.7525 \times 10^{-4}$ & 2.00 & $8.9045 \times 10^{-5}$ & 2.24 & 0.048 \\
\hline$(160,5120)$ & $1.4371 \times 10^{-4}$ & 2.00 & $2.0923 \times 10^{-5}$ & 2.09 & 0.887 \\
\hline$(320,20480)$ & $3.5912 \times 10^{-5}$ & 2.00 & $5.8087 \times 10^{-6}$ & 1.85 & 8.326 \\
\hline
\end{tabular}

We consider the following space-fractional diffusion problem:

$$
\frac{\partial u(x, t)}{\partial t}=c(x) \frac{\partial^{\alpha} u(x, t)}{\partial x^{\alpha}}+s(x, t)
$$

with $0<x<1$ and $0<t \leq 1$.

In order to describe the numerical results, the discrete $l^{2}$-norms, the discrete $l^{\infty}$-norms, and the corresponding convergence rates are defined as follows:

$$
\begin{aligned}
\left\|e^{n}\right\|_{l^{2}} & =\left\|e_{h}^{n}\right\|_{l^{2}}=\left(\sum_{j=1}^{K-1}\left|e_{j}^{n}\right|^{2} h\right)^{1 / 2}, \\
\left\|e^{n}\right\|_{l^{\infty}} & =\max _{1 \leq j \leq K-1}\left|e_{j}^{n}\right|, \\
\text { Rate } & =\frac{\log \left(\left\|e_{h_{1}}^{n}\right\|_{v} /\left\|e_{h_{2}}^{n}\right\|_{v}\right)}{\log \left(h_{1} / h_{2}\right)}, \quad v=l^{2}, l^{\infty},
\end{aligned}
$$

where $e_{j}^{n}=u\left(x_{j}, t_{n}\right)-v_{j}^{n}, n=0,1, \ldots, N-1$, and $h_{1}, h_{2}$ are space mesh sizes.

The discretizing mesh sizes are given according to the following rules.
Case 1. Set $\Delta t=C h^{\alpha}$, where $C$ is a nonnegative integer. By Theorem 7, the convergence order is $O\left(h^{\alpha}\right)$. We get different values of $C$ to verify the theoretical analysis.

Case 2. Set $\Delta t=5 h^{2}$ and take the convergence order is $O\left(h^{2}\right)$.

Two numerical experiments are given with different values of $c(x), \alpha$, and the source function $s(x, t)$. The errors, corresponding convergence rates, and CPU times are listed in the Tables 1-8. The results in Tables $1-8$ are in agreement with the theoretical analysis. In addition, these tables show that, even with very small time-steps, the CPU execution times stay very small as well.

The results in Tables 9-12 are listed by using the finite difference scheme developed by Rui and Huang [19] for Examples 1 and 2. We set $\Delta t=h^{\alpha-0.5}$ and have the same spatial grid sizes $h$ from $1 / 10$ to $1 / 320$, since their error estimates between the numerical and analytical solutions are optimal under the condition $\Delta t=h^{\alpha-0.5}$ for $\alpha \in(1,2)$. Comparing our results in Tables 1-8 with those in Tables 9-12, one can see that the error estimate produced by our method is better than that in [19] in the sense that their convergence rate is $O(h)$. Furthermore, the time-steps in Tables 1-8 are smaller than 
TABLE 4: Error and convergence rates for Example 1.

\begin{tabular}{lccccc}
\hline & & \multicolumn{2}{c}{$\alpha=1.8, \Delta t=5 h^{2}$} & & \\
$(1 / h, 1 / \Delta t)$ & $\|e\|_{l^{\infty}}$ & Rate & $\|e\|_{l^{2}}$ & Rate & - \\
\hline$(10,20)$ & $3.8690 \times 10^{-2}$ & - & $1.3752 \times 10^{-2}$ & 0.001 \\
\hline$(20,80)$ & $9.3129 \times 10^{-3}$ & 2.05 & $2.3787 \times 10^{-3}$ & 2.53 & 0.002 \\
\hline$(40,320)$ & $2.3064 \times 10^{-3}$ & 2.01 & $4.1125 \times 10^{-4}$ & 2.53 & 0.007 \\
\hline$(80,1280)$ & $5.7525 \times 10^{-4}$ & 2.00 & $6.9437 \times 10^{-5}$ & 2.57 & 0.053 \\
\hline$(160,5120)$ & $1.4371 \times 10^{-4}$ & 2.00 & $1.1636 \times 10^{-5}$ & 2.58 & 0.723 \\
\hline$(320,20480)$ & $3.5912 \times 10^{-5}$ & 2.00 & $2.0268 \times 10^{-6}$ & 2.52 & 7.104 \\
\hline
\end{tabular}

TABLE 5: Error and convergence rates for Example 2.

\begin{tabular}{|c|c|c|c|c|c|}
\hline \multicolumn{6}{|c|}{$\alpha=1.6, \Delta t=4 h^{1.6}$} \\
\hline$(1 / h, 1 / \Delta t)$ & $\|e\|_{l^{\infty}}$ & Rate & $\|e\|_{l^{2}}$ & Rate & $\mathrm{CPU}(\mathrm{s})$ \\
\hline$(10,10)$ & $8.1450 \times 10^{-2}$ & - & $2.5828 \times 10^{-2}$ & - & 0.001 \\
\hline$(20,32)$ & $2.3726 \times 10^{-2}$ & 1.78 & $5.3327 \times 10^{-3}$ & 2.28 & 0.001 \\
\hline$(40,92)$ & $8.0849 \times 10^{-3}$ & 1.55 & $1.2920 \times 10^{-3}$ & 2.01 & 0.003 \\
\hline$(80,278)$ & $2.6561 \times 10^{-3}$ & 1.62 & $3.1102 \times 10^{-4}$ & 2.05 & 0.039 \\
\hline$(160,840)$ & $8.7693 \times 10^{-4}$ & 1.60 & $8.2125 \times 10^{-5}$ & 1.92 & 0.131 \\
\hline$(320,2548)$ & $2.8887 \times 10^{-4}$ & 1.60 & $2.5244 \times 10^{-5}$ & 1.70 & 2.193 \\
\hline
\end{tabular}

TABLE 6: Error and convergence rates for Example 2.

\begin{tabular}{|c|c|c|c|c|c|}
\hline \multicolumn{6}{|c|}{$\alpha=1.8, \Delta t=5 h^{1.8}$} \\
\hline$(1 / h, 1 / \Delta t)$ & $\|e\|_{l^{\infty}}$ & Rate & $\|e\|_{l^{2}}$ & Rate & $\mathrm{CPU}(\mathrm{s})$ \\
\hline$(10,14)$ & $5.6493 \times 10^{-2}$ & - & $1.9797 \times 10^{-2}$ & - & 0.001 \\
\hline$(20,44)$ & $1.7108 \times 10^{-2}$ & 1.72 & $5.1683 \times 10^{-3}$ & 1.94 & 0.001 \\
\hline$(40,154)$ & $4.8088 \times 10^{-3}$ & 1.83 & $1.2170 \times 10^{-3}$ & 2.09 & 0.006 \\
\hline$(80,534)$ & $1.3804 \times 10^{-3}$ & 1.82 & $2.9246 \times 10^{-4}$ & 2.06 & 0.058 \\
\hline$(160,1856)$ & $3.9664 \times 10^{-4}$ & 1.80 & $6.9376 \times 10^{-5}$ & 2.08 & 0.456 \\
\hline$(320,6462)$ & $1.1384 \times 10^{-4}$ & 1.80 & $1.6405 \times 10^{-5}$ & 2.08 & 3.441 \\
\hline
\end{tabular}

TABLE 7: Error and convergence rates for Example 2.

\begin{tabular}{|c|c|c|c|c|c|}
\hline \multicolumn{6}{|c|}{$\alpha=1.6, \Delta t=5 h^{2}$} \\
\hline$(1 / h, 1 / \Delta t)$ & $\|e\|_{l^{\infty}}$ & Rate & $\|e\|_{l^{2}}$ & Rate & $\mathrm{CPU}(\mathrm{s})$ \\
\hline$(10,20)$ & $3.8690 \times 10^{-2}$ & - & $1.2540 \times 10^{-2}$ & - & 0.001 \\
\hline$(20,80)$ & $9.3129 \times 10^{-3}$ & 2.05 & $2.2198 \times 10^{-3}$ & 2.50 & 0.002 \\
\hline$(40,320)$ & $2.3064 \times 10^{-3}$ & 2.01 & $4.2601 \times 10^{-4}$ & 2.38 & 0.011 \\
\hline$(80,1280)$ & $5.7525 \times 10^{-4}$ & 2.00 & $8.9084 \times 10^{-5}$ & 2.26 & 0.137 \\
\hline$(160,5120)$ & $1.4372 \times 10^{-4}$ & 2.00 & $2.0611 \times 10^{-5}$ & 2.11 & 0.837 \\
\hline$(320,20480)$ & $3.5921 \times 10^{-5}$ & 2.00 & $5.6286 \times 10^{-6}$ & 1.87 & 7.769 \\
\hline
\end{tabular}

TABLE 8: Error and convergence rates for Example 2.

\begin{tabular}{|c|c|c|c|c|c|}
\hline \multicolumn{6}{|c|}{$\alpha=1.8, \Delta t=5 h^{2}$} \\
\hline$(1 / h, 1 / \Delta t)$ & $\|e\|_{l^{\infty}}$ & Rate & $\|e\|_{l^{2}}$ & Rate & $\mathrm{CPU}(\mathrm{s})$ \\
\hline$(10,20)$ & $3.8690 \times 10^{-2}$ & - & $1.2598 \times 10^{-2}$ & - & 0.001 \\
\hline$(20,80)$ & $9.3129 \times 10^{-3}$ & 2.05 & $2.1751 \times 10^{-3}$ & 2.53 & 0.002 \\
\hline$(40,320)$ & $2.3064 \times 10^{-3}$ & 2.01 & $3.7881 \times 10^{-4}$ & 2.52 & 0.006 \\
\hline$(80,1280)$ & $5.7525 \times 10^{-4}$ & 2.00 & $6.5455 \times 10^{-5}$ & 2.53 & 0.19 \\
\hline$(160,5120)$ & $1.4372 \times 10^{-4}$ & 2.00 & $1.1493 \times 10^{-5}$ & 2.51 & 0.834 \\
\hline$(320,20480)$ & $3.5921 \times 10^{-5}$ & 2.00 & $2.0426 \times 10^{-6}$ & 2.49 & 10.407 \\
\hline
\end{tabular}


TABLE 9: Error and convergence rates for Example 1 by [19].

\begin{tabular}{lcccrc}
\hline & \multicolumn{2}{c}{$\alpha=1.6, \Delta t=h^{1.1}$} & & \\
$(1 / h, 1 / \Delta t)$ & $\|e\|_{l^{\infty}}$ & Rate & & Rate & CPU(s) \\
\hline$(10,14)$ & $5.6493 \times 10^{-2}$ & - & $1.7916 \times 10^{-2}$ & - & 0.001 \\
\hline$(20,28)$ & $2.7238 \times 10^{-2}$ & 1.05 & $6.2433 \times 10^{-3}$ & 1.53 & 0.001 \\
\hline$(40,58)$ & $1.2907 \times 10^{-2}$ & 1.08 & $2.5313 \times 10^{-3}$ & 1.30 & 0.001 \\
\hline$(80,124)$ & $5.9817 \times 10^{-3}$ & 1.11 & $1.1917 \times 10^{-3}$ & 1.09 & 0.015 \\
\hline$(160,266)$ & $2.7764 \times 10^{-3}$ & 1.11 & $6.1784 \times 10^{-4}$ & 0.95 & 0.064 \\
\hline$(320,570)$ & $1.2931 \times 10^{-3}$ & 1.10 & $3.2979 \times 10^{-4}$ & 0.91 & 0.186 \\
\hline
\end{tabular}

TABLE 10: Error and convergence rates for Example 1 by [19].

\begin{tabular}{|c|c|c|c|c|c|}
\hline \multicolumn{6}{|c|}{$\alpha=1.8, \Delta t=h^{1.3}$} \\
\hline$(1 / h, 1 / \Delta t)$ & $\|e\|_{l^{\infty}}$ & Rate & $\|e\|_{l^{2}}$ & Rate & $\mathrm{CPU}(\mathrm{s})$ \\
\hline $\begin{array}{l}(10,20) \\
\text { (1) }\end{array}$ & $3.8690 \times 10^{-2}$ & - & $1.3867 \times 10^{-2}$ & - & 0.001 \\
\hline$(20,50)$ & $1.5013 \times 10^{-2}$ & 1.35 & $5.2908 \times 10^{-3}$ & 1.39 & 0.001 \\
\hline$(40,122)$ & $6.0805 \times 10^{-3}$ & 1.30 & $2.6276 \times 10^{-3}$ & 1.01 & 0.002 \\
\hline$(80,298)$ & $2.4773 \times 10^{-3}$ & 1.30 & $1.3979 \times 10^{-3}$ & 0.91 & 0.019 \\
\hline$(160,734)$ & $1.0038 \times 10^{-3}$ & 1.30 & $7.3133 \times 10^{-4}$ & 0.93 & 0.074 \\
\hline$(320,1806)$ & $5.1822 \times 10^{-4}$ & 0.94 & $3.8051 \times 10^{-4}$ & 0.94 & 1.295 \\
\hline
\end{tabular}

TABLE 11: Error and convergence rates for Example 2 by [19].

\begin{tabular}{|c|c|c|c|c|c|}
\hline \multicolumn{6}{|c|}{$\alpha=1.6, \Delta t=h^{1.1}$} \\
\hline$(1 / h, 1 / \Delta t)$ & $\|e\|_{l^{\infty}}$ & Rate & $\|e\|_{l^{2}}$ & Rate & $\mathrm{CPU}(\mathrm{s})$ \\
\hline$(10,14)$ & $5.6493 \times 10^{-2}$ & - & $1.8277 \times 10^{-2}$ & - & 0.001 \\
\hline$(20,28)$ & $2.7238 \times 10^{-2}$ & 1.05 & $6.1801 \times 10^{-3}$ & 1.56 & 0.001 \\
\hline$(40,58)$ & $1.2907 \times 10^{-2}$ & 1.08 & $2.2795 \times 10^{-3}$ & 1.44 & 0.001 \\
\hline$(80,124)$ & $5.9817 \times 10^{-3}$ & 1.11 & $1.0000 \times 10^{-3}$ & 1.19 & 0.006 \\
\hline$(160,266)$ & $2.7764 \times 10^{-3}$ & 1.11 & $5.1210 \times 10^{-4}$ & 0.97 & 0.071 \\
\hline$(320,570)$ & $1.2931 \times 10^{-3}$ & 1.10 & $2.7682 \times 10^{-4}$ & 0.89 & 0.199 \\
\hline
\end{tabular}

TABLE 12: Error and convergence rates for Example 2 by [19].

\begin{tabular}{|c|c|c|c|c|c|}
\hline \multicolumn{6}{|c|}{$\alpha=1.8, \Delta t=h^{1.3}$} \\
\hline$(1 / h, 1 / \Delta t)$ & $\|e\|_{l^{\infty}}$ & Rate & $\|e\|_{l^{2}}$ & Rate & $\mathrm{CPU}(\mathrm{s})$ \\
\hline$(10,20)$ & $3.8690 \times 10^{-2}$ & - & $1.2610 \times 10^{-2}$ & - & 0.001 \\
\hline$(20,50)$ & $1.5013 \times 10^{-2}$ & 1.37 & $4.2105 \times 10^{-3}$ & 1.29 & 0.001 \\
\hline$(40,122)$ & $6.0805 \times 10^{-3}$ & 1.30 & $1.9787 \times 10^{-3}$ & 1.09 & 0.003 \\
\hline$(80,298)$ & $2.4773 \times 10^{-3}$ & 1.30 & $1.0719 \times 10^{-3}$ & 0.88 & 0.019 \\
\hline$(160,734)$ & $1.0038 \times 10^{-3}$ & 1.30 & $5.7552 \times 10^{-4}$ & 1.57 & 0.086 \\
\hline$(320,1806)$ & $4.4583 \times 10^{-4}$ & 0.95 & $3.0530 \times 10^{-4}$ & 0.91 & 0.633 \\
\hline
\end{tabular}

that in Tables 9-12 with the spatial grid step staying the same. However the CPU execution times increase by a few seconds, which can prove the validity of our presented scheme.

Example 1. The diffusion coefficient $c(x, t)$, the analytical solution $u$, and source coefficient $s(x, t)$ are as follows:

$$
\begin{aligned}
c(x, t) & =1.0, \\
u & =e^{-t} x^{1+\alpha}, \\
s & =-e^{-t}\left(x^{1+\alpha}+\Gamma(2+\alpha) x\right),
\end{aligned}
$$

$$
\begin{aligned}
& u(0, t)=0, \\
& u(1, t)=e^{-t} .
\end{aligned}
$$

The numerical results are listed in Tables 1-4.

Example 2. The diffusion coefficient $c(x, t)$, the analytical solution $u$, and source coefficient $s(x, t)$ are as follows:

$$
c(x, t)=x^{\alpha},
$$




$$
\begin{aligned}
u & =e^{-t} x^{1+\alpha}, \\
s & =-e^{-t} x^{1+\alpha}(1+\Gamma(2+\alpha)), \\
u(0, t) & =0, \\
u(1, t) & =e^{-t} .
\end{aligned}
$$

The numerical results are listed in Tables 5-8.

\section{Conclusion}

In this paper, a uniformly stable explicit finite difference scheme is presented to solve one-dimensional spacefractional diffusion equation. The scheme is constructed combining a WSGD operator with an asymmetric technique. It can be solved explicitly by using different nodal-point stencils at odd and even time-steps. Results of two numerical experiments show that the error estimates are optimal when $\Delta t=O\left(C h^{2}\right)$ and $\Delta t=O\left(C h^{\alpha}\right)$, where $C$ is a nonnegative integer. There may exist super-convergence of the proposed semi-implicit finite difference method, which will be studied in the future.

\section{Data Availability}

The presented data used to support the findings of this study are included with the submitting article.

\section{Conflicts of Interest}

The author declares that they have no conflicts of interest.

\section{Acknowledgments}

This work was supported in part by the Natural Science Foundation of Ningxia (no. NZ17260). I would like to express thanks to Professor Hongxing Rui (Shandong University) for his careful guidance sincerely.

\section{References}

[1] R. Schumer, D. A. Benson, M. M. Meerschaert, and B. Baeumer, "Multiscaling fractional advection-dispersion equations and their solutions," Water Resources Research, vol. 39, pp. 10221032, 2003.

[2] B. Baeumer, D. A. Benson, M. M. Meerschaert, and S. W. Wheatcraft, "Subordinated advection-dispersion equation for contaminant transport," Water Resources Research, vol. 37, no. 6, pp. 1543-1550, 2001.

[3] J. W. Kirchner, X. Feng, and C. Neal, "Frail chemistry and its implications for contaminant transport in catchments," Nature, vol. 403, pp. 524-527, 2000.

[4] M. Raberto, E. Scalas, and F. Mainardi, "Waiting-times and returns in high-frequency financial data: an empirical study," Physica A: Statistical Mechanics and its Applications, vol. 314, no. 1-4, pp. 749-755, 2002.
[5] L. Sabatelli, S. Keating, J. Dudley, and P. Richmond, "Waiting time distributions in financial markets," The European Physical Journal B, vol. 27, no. 2, pp. 273-275, 2002.

[6] M. P. Aghababa and M. Borjkhani, "Chaotic fractional-order model for muscular blood vessel and its control via fractional control scheme," Complexity, vol. 20, no. 2, pp. 37-46, 2014.

[7] W. Meng, D. Yang, and H. Huang, "Prediction of China's sulfur dioxide emissions by discrete grey model with fractional order generation operators," Complexity, vol. 2018, Article ID 8610679, 13 pages, 2018.

[8] M. M. Meerschaert and C. Tadjeran, "Finite difference approximations for fractional advection-dispersion flow equations," Journal of Computational and Applied Mathematics, vol. 172, no. 1, pp. 65-77, 2004.

[9] M. M. Meerschaert and C. Tadjeran, "Finite difference approximations for two-sided space-fractional partial differential equations," Applied Numerical Mathematics, vol. 56, no. 1, pp. 80-90, 2006.

[10] C. Tadjeran and M. M. Meerschaert, "A second-order accurate numerical method for the two-dimensional fractional diffusion equation," Journal of Computational Physics, vol. 220, no. 2, pp. 813-823, 2007.

[11] P. Zhuang, F. Liu, V. Anh, and I. Turner, "New solution and analytical techniques of the implicit numerical method for the anomalous subdiffusion equation," SIAM Journal on Numerical Analysis, vol. 46, no. 2, pp. 1079-1095, 2008.

[12] C. Chen, F. Liu, and K. Burrage, "Finite difference methods and a fourier analysis for the fractional reaction-subdiffusion equation," Applied Mathematics and Computation, vol. 198, no. 2, pp. 754-769, 2008

[13] F. Liu, C. Yang, and K. Burrage, "Numerical method and analytical technique of the modified anomalous subdiffusion equation with a nonlinear source term," Journal of Computational and Applied Mathematics, vol. 231, no. 1, pp. 160-176, 2009.

[14] C. N. Angstmann, I. C. Donnelly, B. I. Henry, B. A. Jacobs, T. A. Langlands, and J. A. Nichols, "From stochastic processes to numerical methods: a new scheme for solving reaction subdiffusion fractional partial differential equations," Journal of Computational Physics, vol. 377, pp. 508-534, 2016.

[15] A. Jhinga and V. Daftardar-Gejji, "A new finite-difference predictor-corrector method for fractional differential equations," Applied Mathematics and Computation, vol. 336, pp. 418432, 2018.

[16] W. Y. Tian, H. Zhou, and W. H. Deng, "A class of second order difference approximations for solving space fractional diffusion equations," Mathematics of Computation, vol. 84, no. 294, pp. 1703-1727, 2015

[17] L. Zhu and H. X. Rui, "Maximum modulus principle estimates for one dimensional fractional diffusion equation," Applied Mathematics-A Journal of Chinese Universities Series B, vol. 30, no. 4, pp. 466-478, 2015.

[18] V. K. Saulyev, Integration of Equations of Parabolic Type by the Methods of Nets, Pergamon Press, New York, NY, USA, 1964.

[19] H. X. Rui and J. Huang, "Uniformly stable explicitly solvable finite difference method for fractional diffusion equations," East Asian Journal on Applied Mathematics, vol. 5, no. 1, pp. 29-47, 2015.

[20] D. J. Evans and A. R. Abdullah, "Group explicit methods for parabolic equations," International Journal of Computer Mathematics. Section A. Programming Theory and Methods. Section B. Computational Methods, vol. 14, no. 1, pp. 73-105, 1983. 
[21] D. J. Evans and A. R. Abdullah, "A new explicit method for the diffusion-convection equation," Computers \& Mathematics with Applications. An International Journal, vol. 11, no. 1-3, pp. 145154, 1985.

[22] B. L. Zhang, "Alternating difference block methods and their difference graphs," Science in China, Series E: Technological Sciences, vol. 41, no. 5, pp. 482-487, 1998.

[23] B. L. Zhang, "Difference graphs of block ADI method," SIAM Journal on Numerical Analysis, vol. 38, pp. 742-752, 2000.

[24] I. Podlubny, Fractional Differential Equations, Academic Press, New York, NY, USA, 1999.

[25] E. Isaacson and H. B. Keller, Analysis of Numerical Methods, vol. 541, John Wiley \& Sons, New York, NY, USA, 1966. 


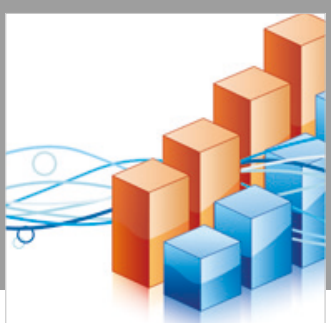

Advances in

Operations Research

\section{-n-m}
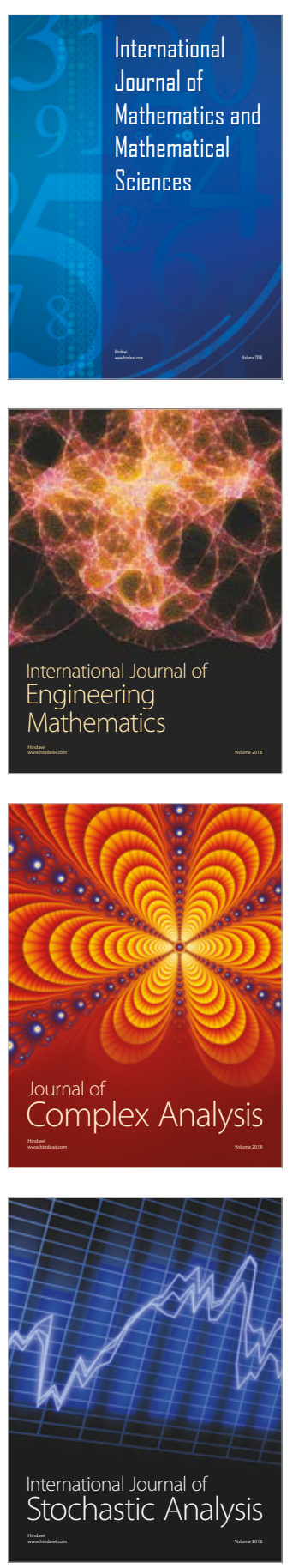
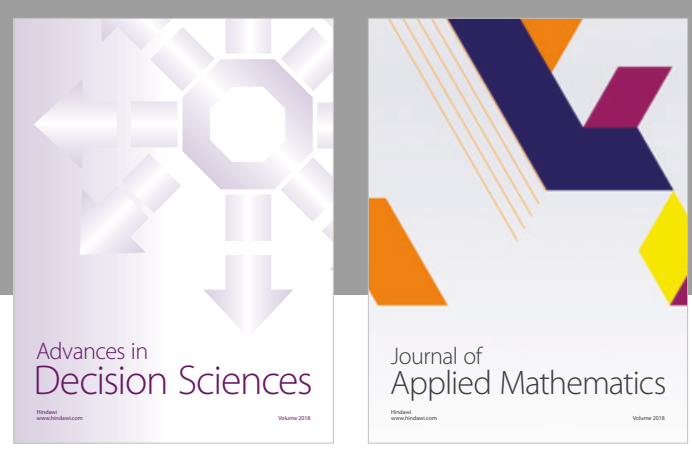

Journal of

Applied Mathematics
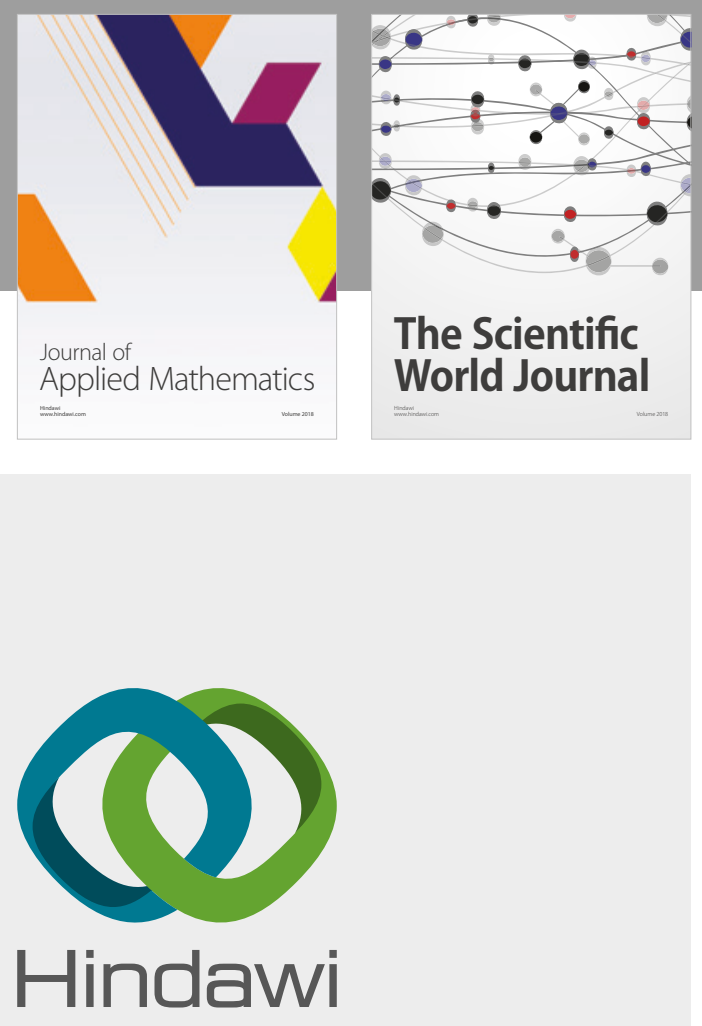

Submit your manuscripts at

www.hindawi.com

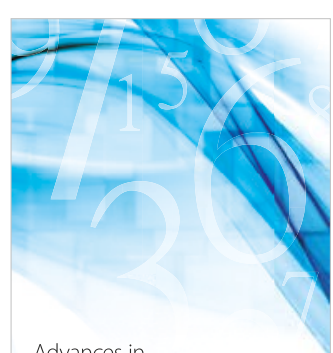

Advances in
Numerical Analysis
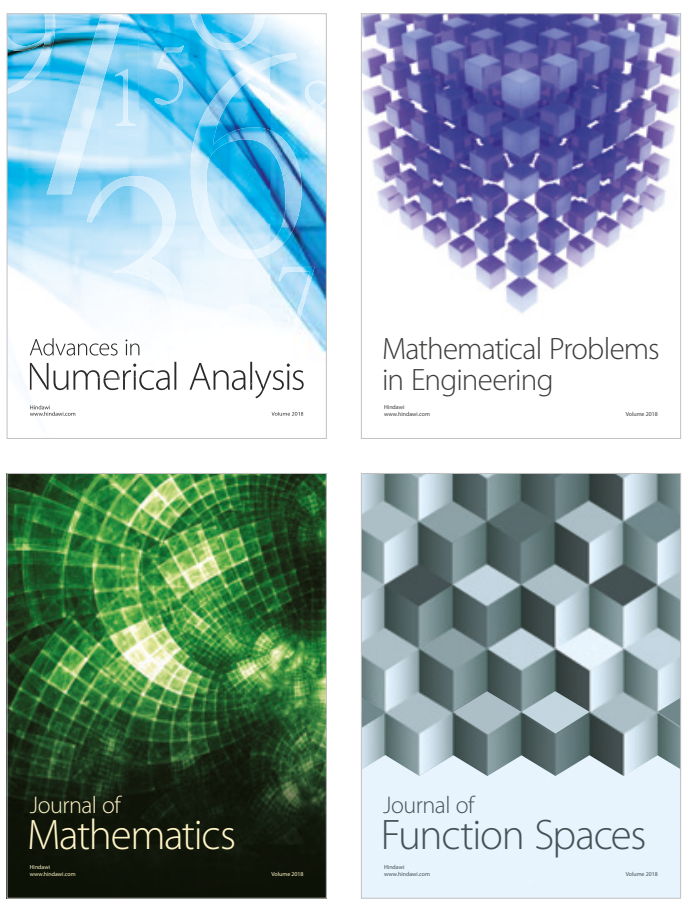

Mathematical Problems in Engineering

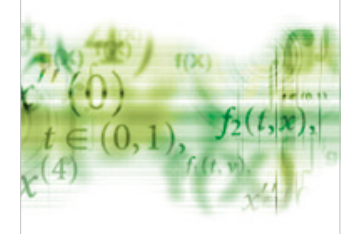

International Journal of

Differential Equations

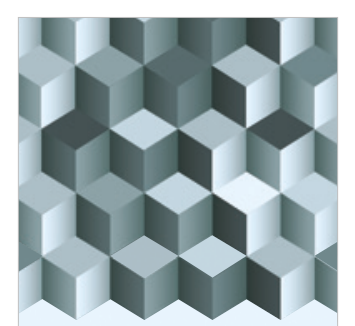

Journal of

Function Spaces
The Scientific

World Journal

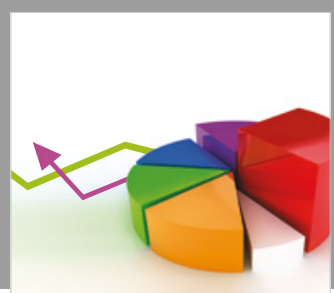

Journal of

Probability and Statistics
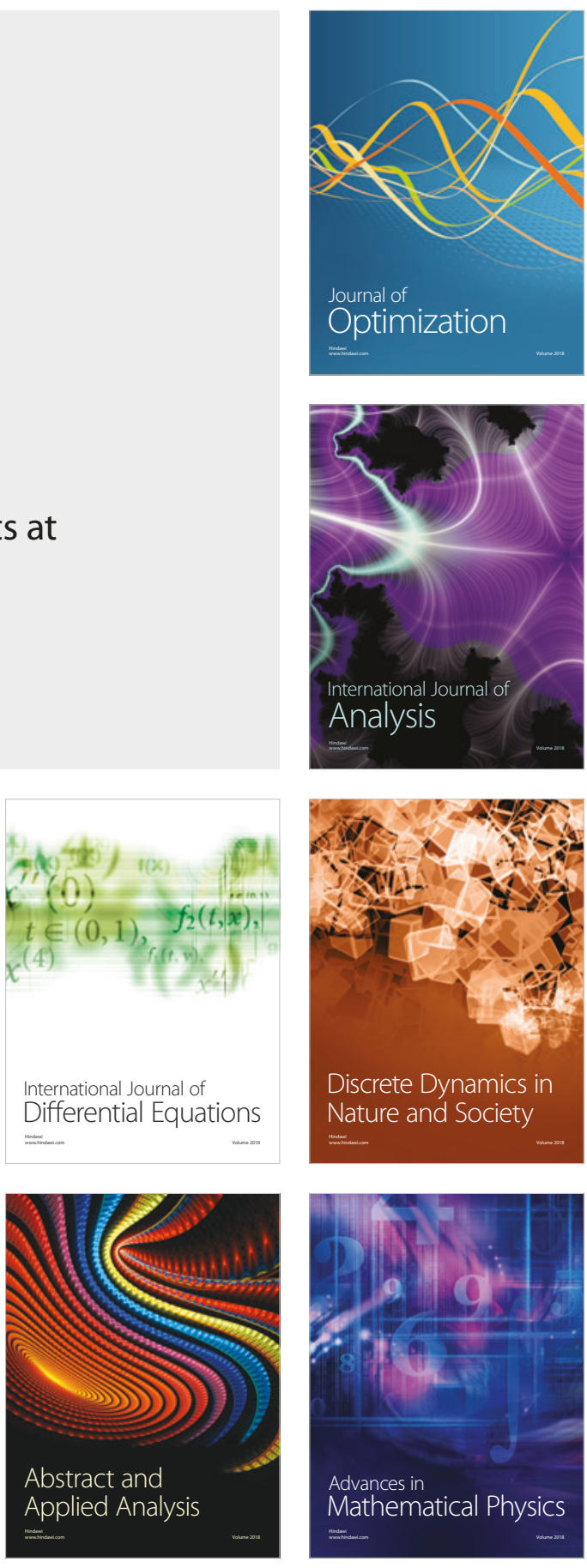